\title{
The three-dimensional Baxter-Wu Model
}

\author{
L. N. Jorge $^{*}$ L. S. Ferreira, ${ }^{\dagger}$ and A. A. Caparica ${ }^{\ddagger}$
}

\begin{abstract}
A classic three-dimensional spin model, based upon the Baxter-Wu scheme, is presented. It is found, by entropic sampling simulations, that the behavior of the energy and magnetization fourthorder cumulants points out to a first order phase transition. A finite-size procedure was performed, confirming that the system scales with the dimensionality $d=3$, and yielding a high-resolution estimate of the critical temperature as $T_{c}=11.377577(39)$.
\end{abstract}

PACS numbers: Valid PACS appear here

The study of three-dimensional spin models in statistical physics have great importance in science of materials, since it can describe, predict, or even design real systems. We can cite the well-know Ising model that is used to describe the Fe-Al magnetic alloy[1-3], once it is arranged in a bcc structure being composed by two interpenetrating cubic lattices. For the disordered case, the phasediagram has been described using the site-diluted spin-1 Blume-Capel model (BC) in a simple cubic arrangement via a mean-field renormalization group approach in the pair approximation[4]. Such technique and model were also used to characterize Fe-Ni-Mn and Fe-Al-Mn alloys [5]. The Ising model is also used to construct metamagnets in thin film geometry models. These systems were studied via Wang-Landau procedure and by importance sampling Monte Carlo (MC) simulations in investigations of their equilibrium phase diagram[6]. Such model was extended to the understanding of non-equilibrium relaxation processes in $\mathrm{Co} / \mathrm{Cr}$ superlattices[7]. Metamagnets compounds $\mathrm{FeCl}_{2}$ and $\mathrm{FeBr}_{2}$ have its properties simulated by a study of a three-dimensional spatially anisotropic Ising superantiferromagnet in the presence of a magnetic field[8], where a rich phase diagram was constructed. There are in nature hexagonal arrangements, as for example, some magnetic systems. Based on MC simulations, Ma et al, have presented results of a film model that is described by a three-dimensional layered honeycomb lattice[9]. This kind of lattice was used by Wang et al in the characterization of molecular based magnetic film $\mathrm{AFe}^{I I} \mathrm{Fe}^{I I I}\left(\mathrm{C}_{2} \mathrm{O}_{4}\right)_{3}[10]$. The most of the hexagonal magnetic materials are described via Ising model in a triangular lattice, as is the case of $\mathrm{Ca}_{3} \mathrm{CO}_{2} \mathrm{O}_{6}$, where the steplike magnetization behavior is strongly dependent of the external field and temperature [11-13]. Hexagonal nanoparticles and nanowires with a core-shell structure, like $\mathrm{CuS} / \mathrm{Cu}_{2} \mathrm{~S}$ with mixed spin $(1 / 2 ; 1)$, and spin-1 $\mathrm{Zn} / \mathrm{Se}$, has been successfully predicted and synthesized [14-20].

\footnotetext{
* Instituto Federal do Mato Grosso - Campus Cáceres, CEP. 78200-000, Cáceres, Mato Grosso. Brazil; lucasnjorge@gmail.com

† Instituto de Física, Universidade Federal de Goiás, C.P. 131 CEP 74001-970, Goiânia, Goiás, Brazil.; lucas.if.ufg@gmail.com

‡ Instituto de Física, Universidade Federal de Goiás, C.P. 131 CEP 74001-970, Goiânia, Goiás, Brazil.; caparica@ufg.br
}

Although there are so many models in hexagonal arrangements in nature or predicted in literature, a threedimensional model via Baxter-Wu interactions is lacking. Therefore the present work aims modeling a threedimensional system that obeys a three-spin interaction like in the two-dimensional Baxter-Wu model, and investigating the order of the phase transition and estimating the subsequent thermodynamic properties, using entropic sampling simulations.

Proposed by Wood and Griffiths[21] in 1972 and exactly solved by Baxter and $\mathrm{Wu}$ [22-24], the Baxter-Wu model is a spin model that considers terms of triple coupling between the spins. It consists in a magnetic system defined on a two-dimensional triangular lattice, where, for the spin- $1 / 2$ case, the spins variables can assume the values $\sigma= \pm 1$ and are located at the vertices of the triangles. The three spin interaction is governed by the Hamiltonian

$$
H_{B W}=-J \sum_{<i, j, k>} s_{i} s_{j} s_{k},
$$

where $J$ is the nearest-neighbor coupling parameter between the spins that fixes the energy scale, and the sum extends over all triangular faces of the lattice.

To construct the three-dimensional version of the Baxter-Wu model, we consider a regular hexagon on the horizontal plane, with six spins in the vertices. In its center, there are three axes crossing it, in such a way that each one can be associated to a hexagon forming an angle of $60^{\circ}$ with the initial plane, as shown in Fig. 1.(a). In this figure we see that the initial hexagon is formed by the dots $(1,2,3,4,5,6)$ and in its center is the zero site. The other three hexagons are formed by $(1,9,8,4,12,10)$, $(2,9,7,5,12,11)$ and $(3,8,7,6,10,11)$. So, a spin, that in the two-dimensional case has six nearest neighbors, and is surrounded by six triangular faces, has in this case 12 nearest neighbors and counts with 24 triangular faces surrounding it.

In this scheme, the three sites that belong to the upper plane, are located above the center of three alternated triangular faces, while the other three in the lower plane are located bellow the centers of the other three triangular faces, as shown in Fig. 1.(a). This lattice is known as hexagonal close-packed (hcp)[25].

Therefore, the three-dimensional Baxter-Wu model is 


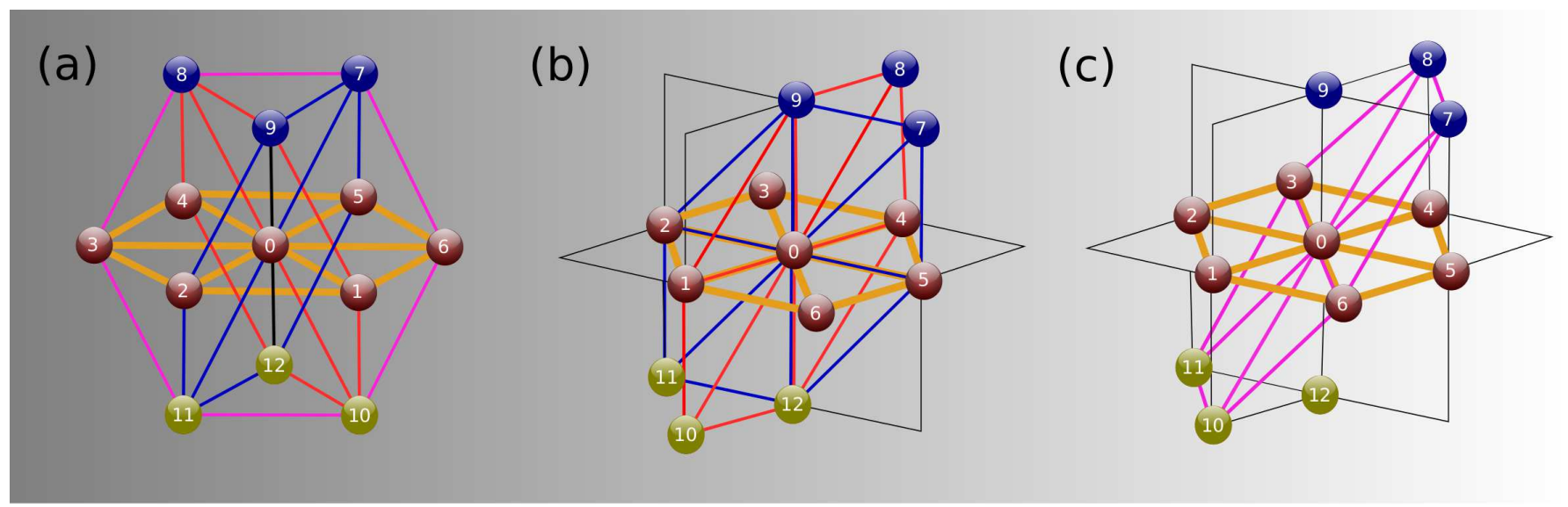

Figure 1. (a) Three-dimensional lattice of the Baxter-Wu model. (b) Three-dimensional lattice of the Baxter-Wu model transposed into a cubic lattice. (c) The oblique plane.

defined in a three-dimensional lattice with triangular interactions, with the energy given by

$$
H_{B W 3 D}=-J \sum_{<i, j, k>} s_{i} s_{j} s_{k}
$$

where the sum extends over all possible triangular faces of the lattice and the spins variables are located at the vertices of the triangles and can assume the integer values $\sigma= \pm 1 . \quad J$ is the constant that scales the energy of the lattice, being the same in all directions. Unlike the two-dimensional version of the model, which displays four ground state configurations - one ferromagnetic and three ferrimagnetic - the 3D Baxter-Wu model has a single ground state configuration, namely the ferromagnetic one. When we try to construct a three-dimensional ferrimagnetic configuration, the triangular faces are satisfied for two planes, but for the third and fourth planes, frustrations appear, showing that it is impossible to obtain such ferrimagnetic constructions in three dimensions.

The Hamiltonian may then be decomposed in sums over four planes

$$
\begin{aligned}
H= & -J\left[\sum_{<i, j, k>} s_{i} s_{j} s_{k}\right]_{X Y}-J\left[\sum_{<i, j, k>} s_{i} s_{j} s_{k}\right]_{X Z} \\
& -J\left[\sum_{<i, j, k>} s_{i} s_{j} s_{k}-J\left[\sum_{Y Z} s_{i} s_{j} s_{k}\right]_{O b l . j, k>},\right.
\end{aligned}
$$

which extend over all triangles of the lattice in each plane $X Y, X Z, Y Z$ (Fig. 1.(b)) and the oblique plane (Fig. 1.(c)), respectively.

In the cubic scheme, the energy of a particular configuration is given by

$$
\begin{aligned}
& E=\frac{J}{3}\left[\sum _ { i = 1 } ^ { L } \sum _ { j = 1 } ^ { L } \sum _ { k = 1 } ^ { L } s _ { i , j , k } \left(s_{i+1, j, k} s_{i, j-1, k}+s_{i, j-1, k} s_{i-1, j-1, k}+s_{i-1, j-1, k} s_{i-1, j, k}+s_{i-1, j, k} s_{i, j+1, k}+\right.\right. \\
& \left.s_{i, j+1, k} s_{i+1, j+1, k}+s_{i+1, j+1, k} s_{i+1, j, k}\right) \\
& \quad+\sum_{i=1}^{L} \sum_{j=1}^{L} \sum_{k=1}^{L} s_{i, j, k}\left(s_{i+1, j, k} s_{i, j, k+1}+s_{i, j, k+1} s_{i-1, j, k+1}+s_{i-1, j, k+1} s_{i-1, j, k}+s_{i-1, j, k} s_{i, j, k-1}+s_{i, j, k-1} s_{i+1, j, k-1}+\right. \\
& \left.s_{i+1, j, k-1} s_{i+1, j, k}\right) \\
& \quad+\sum_{i=1}^{L} \sum_{j=1}^{L} \sum_{k=1}^{L} s_{i, j, k}\left(s_{i, j, k+1} s_{i, j-1, k}+s_{i, j-1, k} s_{i, j-1, k-1}+s_{i, j-1, k-1} s_{i, j, k-1}+s_{i, j, k-1} s_{i, j+1, k}+s_{i, j+1, k} s_{i, j+1, k+1}+\right. \\
& \left.s_{i, j+1, k+1} s_{i, j, k+1}\right) \\
& \quad+\sum_{i=1}^{L} \sum_{j=1}^{L} \sum_{k=1}^{L} s_{i, j, k}\left(s_{i-1, j-1, k} s_{i-1, j, k+1}+s_{i-1, j, k+1} s_{i, j+1, k+1}+s_{i, j+1, k+1} s_{i+1, j+1, k}+s_{i+1, j+1, k} s_{i+1, j, k-1}+\right. \\
& \left.\left.s_{i+1, j, k-1} s_{i, j-1, k-1}+s_{i, j-1, k-1} s_{i-1, j-1, k}\right)\right]
\end{aligned}
$$

where the division by three is because in this sums each triangular face is counted three times.

In this work we adopted the order parameter as the total magnetization of the system, $M=\sum_{i, j, k=1}^{L} s_{i, j, k}[26-$
28], thus, in the simulations we picked only non-multiple of three lattice sizes.

The entropic simulations applied to our model are based on the Wang-Landau method[29], that by means 

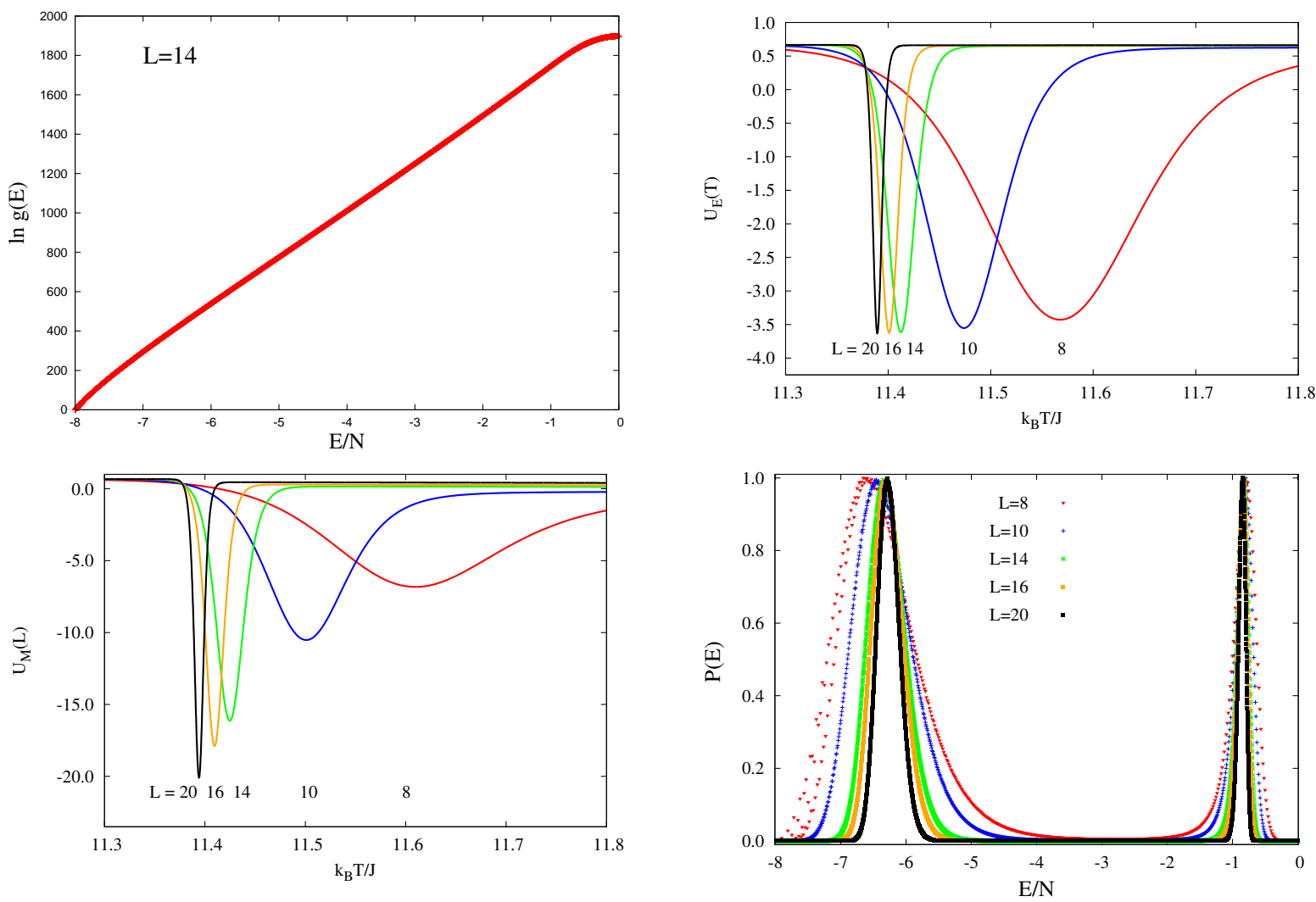

Figure 2. (Top left) Logarithm of the density of states for the $L=14$. (Top right) Fourth-order energy cumulants as funtions of temperature. (Bottom left) Fourth-order magnetization cumulants as functions of temperature. (Bottom right) Energy probability distributions as function of the energy per particle.

of a random walk in energy space allows the construction of the density of states $g(E)$, generating a flat histogram for the energy distribution, and then the estimation of the canonical averages of any thermodynamic quantities. In our simulations we include some improvements that enhance the accuracy and lead to substantial savings in CPU time. Namely, (i) we adopt the Monte Carlo sweep before updating the density of states, avoiding taking into account highly correlated configurations, (ii) we begin to accumulate the microcanonical averages only from the eighth Wang-Landau level $\left(f_{7}\right)$, such that we discard the initial configurations that do not match with those of maximum entropy[30], (iii) we use a checking parameter $\varepsilon$ for halting the simulation[31] (the computational process is halted if the integral of the specific heat over a range of temperature calculated with the current density of states during the simulations varies less then $10^{-4}$ during a whole Wang-Landau level), and (iv) we begin all simulations, for all lattice sizes, beginning from the outputs of a single run up to the Wang-Landau level $f_{6}$, because up to this point the current density of states is not biased yet and can proceed to any final result that would be obtained beginning from the first Wang-Landau level $f_{0}$ [32], a procedure that allows saving about $60 \%$ of CPU time.

We carried out entropic simulations for $L \times L \times L$ lattices, picking $L=8,10,14,16$ and 20, with $n=$ $24,20,20,16$ and 16 independent runs, respectively. We performed independent simulations on five such sets, so that all our final results and error bars are taken as averages over the results of these sets.

In Fig. 2.(a) we show the logarithm of the density of states of the lattice size $L=14$.

The behavior of the fourth-order energy and magnetization cumulants

$$
U_{X}(L)=1-\frac{\left\langle X^{4}\right\rangle}{3\left\langle X^{2}\right\rangle^{2}}, \quad X \equiv E, M
$$

shown in Figs. 2.(b) and 2.(c) give us solid evidences that our novel model undergoes a 1st order phase transition. The energy cumulants intersect at a point close to the transition temperature, while the magnetization cumulants exhibit sharp inverted minima, as expected in a discontinuous phase transition. In addition, the energy probability distributions display double peaks of same high at the finite-size transition temperature, with a null probability valley between them, as we see in Fig. 2.(d).

In a system that suffers a discontinuous phase transition it is expected that the maxima of the specific heat and the magnetization should scale with the dimensionality. Another quantity that displays the dimensionality of the system in a discontinuous phase transition is $1 / \nu$ 

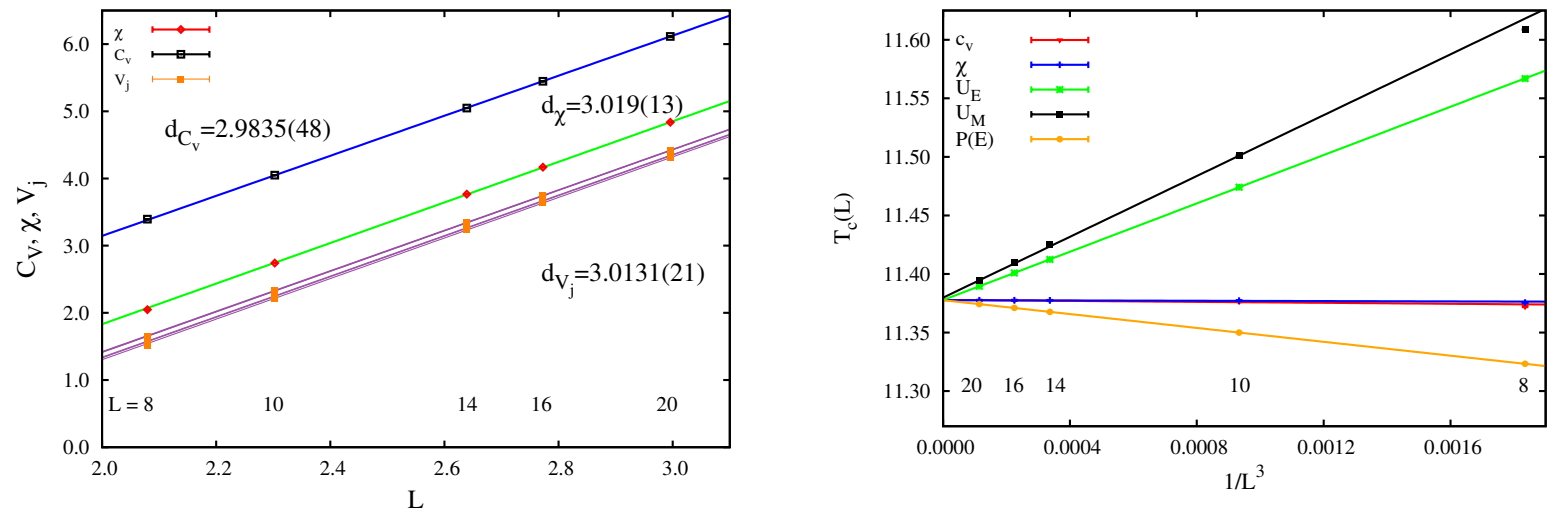

Figure 3. (Left) Log-log plot of the maxima of the specific heat, the susceptibility, and the cumulants $V_{j}$ with the lattice size. The linear coefficients are close to the dimensionality of the system. (Right) Dependence on temperature of the minima of the energy and magnetization cumulants, the maxima of the specific heat and the susceptibility, and the double peaks of same high of energy density of probability, against $1 / L^{3}$.

in $[26,33-35]$

$$
V_{j} \approx \frac{1}{\nu} \ln L+\mathcal{V}_{j}\left(t L^{\frac{1}{\nu}}\right)
$$

In Fig. 3 (left) we present this finite-size scaling behavior for the first of the five sets simulated. The values of all samples are shown in the first three columns of Table I, where the final averages are given at the last line. As usual in these entropic sampling procedures we neglect the error bars and calculate the averages using the central values[26, 31, 32]. In order to obtain our final results

\begin{tabular}{cccc}
\hline \hline$d_{C_{v}}$ & $d_{\chi}$ & $d_{V_{j}}$ & $T_{c}$ \\
\hline $2.9835(48)$ & $3.019(13)$ & $3.0131(21)$ & $11.377618(30)$ \\
$2.9813(48)$ & $3.020(11)$ & $3.0140(22)$ & $11.377486(41)$ \\
$2.9810(45)$ & $3.018(11)$ & $3.0141(22)$ & $11.377690(17)$ \\
$2.9815(44)$ & $3.024(12)$ & $3.0154(23)$ & $11.377600(34)$ \\
$2.9829(40)$ & $3.017(11)$ & $3.0135(20)$ & $11.377489(36)$ \\
\hline $2.98205(50)$ & $3.0197(12)$ & $3.0140(10)$ & $11.377577(39)$ \\
\hline \hline
\end{tabular}

Table I. Five finite-size scaling results yielding exponents close to the dimensionality for the maxima of specific heat, the susceptibility, and the cumulants $V_{j}$, and for the critical temperature. The averages over all runs are displayed at the last line.

for $d$, the dimensionality, we again neglect the error bars and take an average of the three final results, yielding $d_{C_{v}, \chi, V_{j}}=3.005(12)$.

According to Fisher and Berker [36], in first order transitions all finite size scaling procedures are made in terms of powers of the lattice size, $L^{-d}$. Once confirmed that the system scales with the dimensionality, we can proceed with the determination of the critical temperature as the extrapolation for $L \rightarrow \infty\left(L^{-d}=0\right)$ of the best linear fits of the temperatures of the maxima of the specific heat and the susceptibility, the minima of the energy and magnetization fourth-order cumulants, and the temperatures where the energy probability distribution displays double peaks of same high. In Fig. 3 (right) we depict these best fits for the first set of simulations. The mean critical temperatures for each set are displayed in Tab. I, with the best estimate appearing in the last line, yielding $T_{c}=11.377577(39)$. This new three-dimensional model may consist of an useful platform for the simulation of existing compounds in nature.

\section{ACKNOWLEDGMENTS}

We acknowledge the computer resources provided by LCC-UFG and IF-UFMT. L. N. Jorge and L. S. Ferreira acknowledge the support by FAPEG and CAPES, respectively.
[1] D. Dias, J. R. de Sousa, and J. Plascak, Physics Letters A 373, 3513 (2009).

[2] J. A. Plascak, L. E. Zamora, and G. A. Pérez Alcazar, Phys. Rev. B 61, 3188 (2000).

[3] A. Freitas, D. de Albuquerque, and N. Moreno, Physica A: Statistical Mechanics and its Applications 391, 6332 (2012).
[4] D. Dias and J. Plascak, Physics Letters A 375, 2089 (2011).

[5] D. Peña Lara, G. A. Pérez Alcázar, L. E. Zamora, and J. A. Plascak, Phys. Rev. B 80, 014427 (2009).

[6] Y.-L. Chou and M. Pleimling, Physical Review B 84, 134422 (2011).

[7] T. Mukherjee, M. Pleimling, and C. Binek, Physical Re- 
view B 82, 134425 (2010).

[8] O. D. Salmon, M. A. Neto, J. R. Viana, I. T. Padilha, and J. R. de Sousa, Physics Letters A 377, 1991 (2013).

[9] B. Ma and W. Jiang, IEEE Transactions on Magnetics 47, 3118 (2011).

[10] W. Wang, W. Jiang, D. Lv, and F. Zhang, Journal of Physics D: Applied Physics 45, 475002 (2012).

[11] A. Maignan, V. Hardy, S. Hebert, M. Drillon, M. R. Lees, O. Petrenko, D. M. K. Paul, and D. Khomskii, J. Mater. Chem. 14, 1231 (2004).

[12] A. Maignan, C. Michel, A. Masset, C. Martin, and B. Raveau, The European Physical Journal B-Condensed Matter and Complex Systems 15, 657 (2000).

[13] Y. B. Kudasov, Physical review letters 96, 027212 (2006).

[14] U. K. Rößler and A. N. Bogdanov, Journal of magnetism and magnetic materials 269, L287 (2004).

[15] W. Wang, D.-d. Chen, D. Lv, J.-p. Liu, Q. Li, and Z. Peng, Journal of Physics and Chemistry of Solids (2017).

[16] D. Lv, F. Wang, R.-j. Liu, Q. Xue, and S.-x. Li, Journal of Alloys and Compounds (2017).

[17] T. Kaneyoshi, physica status solidi (b) 248, 250 (2011).

[18] R. Masrour, A. Jabar, A. Benyoussef, M. Hamedoun, and L. Bahmad, Physica B: Condensed Matter 472, 19 (2015).

[19] J. Ahmed, S. Sharma, K. V. Ramanujachary, S. E. Lofland, and A. K. Ganguli, Journal of colloid and interface science 336, 814 (2009).

[20] Y.-B. Chen, L. Chen, and L.-M. Wu, Crystal Growth and Design 8, 2736 (2008).

[21] D. Wood and H. Griffiths, Journal of Physics C: Solid
State Physics 5, L253 (1972).

[22] R. J. Baxter and F. Wu, Physical Review Letters 31, 1294 (1973).

[23] R. Baxter, Australian Journal of Physics 27, 369 (1974).

[24] R. Baxter and F. Wu, Australian Journal of Physics 27, 357 (1974).

[25] N. W. Ashcroft and D. N. Mermin, Solid State Physics, edited by D. G. Crane (Saunders College Publishing, 1976).

[26] L. N. Jorge, L. S. Ferreira, S. A. Leão, and A. A. Caparica, Brazilian Journal of Physics 46, 556 (2016).

[27] M. Santos and W. Figueiredo, Physical Review E 63, 042101 (2001).

[28] I. Velonakis and S. Martinos, Physica A: Statistical Mechanics and its Applications 392, 2016 (2013).

[29] F. Wang and D. P. Landau, Physical Review E 64, 056101 (2001)

[30] A. A. Caparica and A. G. Cunha-Netto, Phys. Rev. E 85, 046702 (2012).

[31] A. A. Caparica, Phys. Rev. E 89, 043301 (2014).

[32] L. Ferreira, L. Jorge, S. Leão, and A. Caparica, Journal of Computational Physics 358, 130 (2018).

[33] A. M. Ferrenberg and D. P. Landau, Phys. Rev. B 44, 5081 (1991).

[34] A. A. Caparica, A. Bunker, and D. P. Landau, Phys. Rev. B 62, 9458 (2000).

[35] A. Caparica, S. A. Leão, and C. J. DaSilva, Physica A: Statistical Mechanics and its Applications 438, 447

[36] M. E. Fisher and A. N. Berker, Physical Review B 26, 2507 (1982) 